[A prepring, refer to with consent from the author]

\title{
Unblurring the Boundary Between Daily Life and Gameplay in Location-based Mobile Games, Visual Online Ethnography on Pokémon GO
}

\author{
Paula Alavesa $^{1}$ (Corresponding author) and Yueqiang $\mathrm{Xu}^{2,3}$ \\ ${ }^{1}$ Center for Ubiquitous Computing, University of Oulu, Finland \\ P.O Box 4500, Oulu, FI-90014, Finland \\ Email:paula.alavesa@oulu.fi
}

${ }^{2}$ Martti Ahtisaari Institute of Global Business and Economics, University of Oulu, Finland

P.O Box 4600, FI-90014, Finland

${ }^{3}$ Nokia Networks, Oulu, Finland

Kaapelitie 4, 90650 Oulu, Finland

Email: yueqiang.xu@oulu.fi 


\section{ABSTRACT}

Observing blending of realities, daily life and gameplay in location-based mobile games is challenging. This study aims at observing this blending by targeting a vast number of images $(\mathrm{N}=2432)$, which have been taken during gameplay of a well-known game, Pokémon GO. Images were collected from social media communities of Pokémon GO players in Twitter, Facebook, and Instagram, and analyzed using visual and online ethnography. To keep the sample size manageable for analysis the images were collected only from Nordic Pokémon GO player communities in eight cities during 2016-2018. The findings show that blending of daily life and gameplay are observable from the shared photos especially from the augmented reality screenshots which is why in this article the context of gameplay, both outdoors and indoors, in Pokémon GO is described in more detail than in previous studies.

KEYWORDS: Location-based mobile games, social media, visual ethnography 


\section{Introduction}

Pervasive games are games expanding what is traditionally considered gaming. While early $21^{\text {st }}$ century these games were still marginal, today some of its subcategories such as location-based mobile games (LBMGs) have become mainstream. The context of gameplay, the city streets, is an integral part of LBMGs. How the gameplay is entwined with daily life, is an equally important characteristic (Liu, Wagner \& Suh, 2017; Montola Stenros, \& Waern, 2009; Paavilainen et al., 2017). For research, difficulties posed by the uncontrolled environment, create challenges for observing the blended realities of game space and blending of daily life and gameplay as it happens.

Despite the decline in number of players, Pokémon GO still has a sizable global player community and the game has been actively updated to keep the players' interests alive (Niantic, 2016). This player base and its behavior offers rich pool of material for analyzing game context and blending of realities during gameplay of LBMGs. Although Pokémon GO can be characterized as an "an augmented reality (AR) game", the AR feature is not extensively used or is seldom required in gameplay (Alha, Koskinen, Paavilainen, \& Hamari, 2019; Azuma, 1997; Paavilainen et al., 2017). The blending of game's digital contents augments the physical environment in a way that the game can be characterized as a pervasive game, more specifically a mixed reality or a hybrid reality game in addition to being a LBMG (Benford \& Giannachi, 2011; Montola et al., 2009; de Souza e Silva, 2009).

Empirical research on Pokémon GO often utilizes ethnographic and survey methods (Ishii, Ajito \& Kawahata, 2016; Liu et al. 2017; Paasovaara, Jarusriboonchai \& Olsson, 2017; Paavilainen et al., 2017; Vella et al., 2017). These approaches can produce knowledge either on the gameplay primarily in the physical environment or, as it is with surveys, information that is asynchronous to 
the gameplay. Ishii et al. (2016) state that a key problem is that Pokémon GO players do not always post to social media during gameplay. In this study visual online ethnography and a qualitative analysis of 2432 images from social media sites (Facebook, Instagram, Twitter) of Pokémon GO communities from eight Nordic cities was conducted. The images are taken while playing the game, therefore, despite there may be delays when posting them, the imagery makes the blending of daily life and gameplay perceivable as it happens.

This article first introduced the related work on the significance of social networking for Pokémon GO players including introduction on relevant theories on how playful spaces in cities have been described. How AR is used in Pokémon GO gameplay is in addition explained following with introduction to the method used in this study, visual online ethnography. Results and their relevance in the light of introduced related work and theories are discussed before concluding the article.

\section{Literature review}

The global launch of Pokémon GO in July 2016 set foundation for social connectedness. Participating in the hype became a shared cultural experience that created a sense of belonging in connection to both people and places (Vella et al., 2017). There has been a similar rush of interest by researchers, as over the past years close to ten thousand academic publications have been written from various perspectives (Google Scholar, search phrase "Pokémon GO"). Although the game elements that encourage exploration are key reasons for continued Pokémon GO gameplay (Alha et al., 2019). Pokémon GO also has social implications. 90\% of the players play with people they know (e.g. family members from different generations who are brought closer by the shared experience). "Friendship maintenance" has been found to be one of the key motivators for playing the game (Vella et al., 2017). The players state that the game does act as an icebreaker between 
strangers and shared goals during gameplay enhance social cohesion (Paavilainen et al., 2017; Vella et al., 2017; Yang \& Liu, 2017). However, this type of social cohesion is mostly observed in the physical world, outdoors while in gameplay. Wang et all. (2018) state that it is possible that cyber-physical social networks provide social support different purely physical or virtual social networks. The enhanced social cohesion does indeed span to the online communities. Many players state that they have received unexpected support from their Facebook team members while injured or sick (Paavilainen et al., 2017; Vella et al. 2017). Pokémon GO players form an online community around the gameplay, where the overarching theme of discussion is the game itself. Game achievements and captured pokémon are reported extensively, when personal matters, prevalent in personal social media content, are not as common (Hu, Manikonda \& Kambhampati, 2014; Majgaard \& Larsen, 2017). Activity in social networks has been found to result in enhanced physical activity in related activities, which combined with the increased physical activity of the Pokémon GO players can enhance the healthy effect (Althoff, Jindal \& Leskovec, 2017; Althoff, White \& Horvitz, 2016). The reach of Pokémon GO to the digital space of social media also has other implications. For instance, Pokémon GO has been found to revive lost interest in natural history and nature conservation. A Twitter hashtag \#Pokeblitz helps people identify "real" species photographed while playing (Brulliard 2016; Dorward, Mittermeier, Sandbrook \& Spooner, 2017).

\subsection{Context of gameplay}

Spatial expansion is a key quality of pervasive games as defined by Montola (2005). This expansion refers to the gameplay taking place in a setting or expanding in scope outside traditional game arenas; the gameboard metaphor is often used when describing this expansion: instead using a game board, the game is played on the city streets (Montola et al., 2009) and there may be a bias towards assuming that pervasive games are outdoor games. The context for many current pervasive 
games (Southerton, 2013) and hybrid reality games (de Souza e Silva, \& Sutko, 2008; de Souza e Silva, 2009; 2016) is often urban as the urban environment provides social and technological possibilities for the games. Some location-based mobile games (LBMGs) are, for instance Zombies run! (Southerton, 2013) a popular pervasive exergame, specifically designed to be played outdoors. Other games, such as Pokémon GO (Niantic., 2016), do not directly restrict the game context and as of spring 2020, due to the COVID-19 pandemic, the game has been updated to support indoor play better. Although locationing on mobile phones does not work as well indoors as it does outdoors, there are many game activities that do not require precise locationing or locationing at all.

What current LBMGs have done is expanding the context of gameplay outside and around the physical hubs, such as pervasive displays, that have been previously used for gameplay in urban areas (O'Hara, Glancy, \& Robertshaw, 2008). The games are permeating contexts that in theory were early $21^{\text {st }}$ century considered playful, but then there were no singular games that would have been able to pervade the whole strata of different possible contexts for gameplay. This is what some of the current LBMGs, such as Pokémon GO can do. There are many perspectives to what extend cities are playful. Alavesa (2018) suggests in her work alternate mediators for different layers of urban space, one of those mediators are the affordances created by games. Sicart (2016) takes this further and suggests play itself as the interface that allows interaction with city spaces that are defined not just by architectonic fixtures. He states that this playful interaction is not only based on human playfulness (Huizinga, 1955), but something that can and should be fostered by the cities. Inspired by Sicart's (2016) perspective Wong, Balmford, Hjorth \& Richardson (2019, p 266) suggest that "Through the lens of playful media, we can gain new insights into how "work" or "home" are negotiated, especially as we move through different places and spaces." According 
to Sicart (2014) almost any space can become a playground. Spaces encompass cues, which signal players that they are available for play i.e. playful spaces. There is however a lack of discussion on how the signals are interpreted or what they can be when the spaces can be any spaces in urban environment and not areas staged for play by artificial situations, norms, rules and objects. The digital playgrounds can be situated fully in virtual worlds, however there is another kind of hybrid playground that combines the blended digital contents, such as Pokémon, and the physical spaces (Alavesa, 2018; Sicart, 2014; de Souza e Silva, 2009; Wang et al. 2018). In Pokémon GO many of the so-called artificial objects defining the playfulness of a space are in fact digital and globally distributed to points of interest mainly in urban areas (Colley et al. 2017). Many of the previous mappings of play in the cities encompass the whole city without going into details.

\subsection{Hybrid reality and extending across digital and physical spaces}

For this study, hybrid reality logic (de Souza e Silva., 2009; de Souza e Silva., 2016; de Souza e Silva \& Sutko 2008) is adopted. It states that LBMGs combine different realities such as digital environments, social networks and the city streets. There are many examples of hybrid spaces or realities that span and entwine digital and physical in Pokémon GO. Global simultaneous grouping of people with a shared goal of catching pokémon forms an "information ground". This is a situation where people group with a purpose other than information sharing yet flow of information occurs as the participants interact (Ishii et al., 2016; Pettigrew, 1999). This phenomenon forms the basis of the study of Lee, Windleharth, Yip \& Schmalz (2017) on the information behavior of Pokémon GO players. They noticed in their ethnographical study that in the physical environment people share information directly, shouting or physically moving between locations. Simultaneously in the online space, the information flow is comprised of indirect observations such as checking game status (e.g. Pokestop ownership) or weather information. They state that 
they failed to observe blending of the information spaces, although they describe how the players use online communities to organize and disseminate knowledge (Lee et al., 2017). Wang et all. (2018) deepen the observations into this social networking by combining spatial analysis and ethnographical surveys on the street of Melbourne, Australia to attain results from social networks on the street in relation to game specific locations. They call the blending of realities in Pokémon GO as "cyber-physical social networks". Their study was restricted to hours considered free time; hence the full blending of daily life and gameplay was outside the scope of their study.

It can be argued that the urban game arena as a hybrid information ground contains both the physical and the online communities where the flow of information reaches. This study also relies on Sicart's (2016) theory or what he calls rhetoric of play, which embraces the notion of ambiguity of play in addition to accepting the cities as technocultural landscapes comprised of cultural, social and technical. While hybrid reality logic (de Souza e Silva., 2009) describes the inseparable entanglement of the physical, digital and social in urban environments. Following these exhaustive theories, the focus of this inspection is targeted where the entanglement of playful, digital, physical and social can be seen, the (AR) snapshots and photos the players publish in online communities.

\subsection{AR use in Pokémon GO}

Though Pokémon GO is characterized as AR game, the AR features are not necessary for gameplay. Paavilainen et al. (2017) report in their extensive study that only $9 \%$ play with AR on while $75,1 \%$ play AR mode off, and the rest switch between the modes. In fact, the AR mode does not offer any benefits to gameplay. Although some players state that the use of technology motivates them to play (Alha et al., 2019). The use of AR consumes battery as it requires the back camera of the smart phone to be turned on. In addition, the pokémon may disappear from camera view when one moves the phone, which can be frustrating for players. From the perspective of the 
player experience, it also seems that the AR functionality is not necessary for spatial or sensory immersion, both of which are high ( $88 \%$ and $72 \%)$ for players of whom minority are using the AR (Liu et al., 2017; Paavilainen et al., 2017).

\section{Methodology}

In this study visual online ethnography (Kozinets, 2015; Lotay, 2016; Schwartz, 1989) was used to distill knowledge from the AR and other images players post in Pokémon GO related social media sites (Twitter, Facebook, Instagram).

Ethnography is derived from anthropological tradition of documenting and interpreting aspects of human experience and cultural behavior. According to Senior and Chenhall (2012), ethnography is a methodology with a non-reductionist view of the world (Althoff et al., 2017), capable of capturing the lived experiences of human, their context to gain in depth understanding. Skågeby (2008) defines ethnography as a description of individuals, groups or cultures in their own environment often over an extended period. Its advantage arises from the first-hand witnessing and discovering which is seen as central to the proper understanding and interpretation of a specific context of action and subsequent actions (Skågeby, 2008). The study presented in this article was conducted in a unique time-period in Pokèmon GO's evolution, since of February 2019 and in many updates thereafter the use of AR feature in Pokémon GOhas been enhanced, which is why there is now an abundance of AR images in social media, and it is not at all certain if the image has been taken for the purpose of taking a snapshot with a specific pokémon or while playing the game. The current version of the game does at time also force the use of AR to complete game tasks, which influences the spontaneity of available imagery.

Expanding from the conventional field-based ethnography approach, digital ethnography is unique in that it is an ethnography in, of, and through the web medium with ubiquity (Kozinets, 2015). 
Various scholars suggest that online ethnography draws much on the procedures and considerations of traditional ethnography (Hine, 2000; Skågeby, 2008). Online ethnography is "traditional ethnography" adapted to the study of online communities. Online digital ethnography involves several data collection methods, such as document collection and online observation (Kozinets, 2015; Lotay, 2016). In the case of this study, it is the observationin several Pokémon GO related online communities.

To fully understand how players interact with the hybrid realities that are brought forward by Pokémon GO, it is necessary to take into account the individual and socio-cultural meanings of player-behavior and activities on social media, which cannot be fully captured without attention to the context in which they are situated (Androutsopoulos, 2008). In this study, visual online ethnography was carried out by analyzing extensive number of images from online player communities. Similar approach has been utilized with visual and other digital contents in previous research on Facebook groups (Beaulieu, 2004; Hine, 2000; Markham \& Baym, 2008; Pittman \& Reich, 2016).

Photographs themselves are not just messages that trigger objective existing meaning in the viewer. The process of image making, the photographic methods, enrich the images with meaning (Schwartz, 1989). The environmental images (Lynch, 1960) taken by the Pokémon GO players combine both perception and the experience of the physical environment, which are filtered by action, in this case gameplay. Despite offering only a glimpse into the Pokémon GO players' lives using a method of observing the online community offers advantages in being able to expand the scope of the observation geographically and socially (Griffiths, Lewis, de Gortari \& Kuss, 2016). As Madden (2014) argues, ethnography's defining feature is not in face-to-face contact but "a 
shared sense of participation", which is methodologically important for this study to be called ethnography.

\section{Material and data analysis}

The study was initiated with a mapping study on literature related to Pokémon GO. In addition, the game was played by the observers to familiarize themselves with the gameplay and support understanding the images. The literature related to Pokémon GO was searched with Google Scholar and search phrase "Pokémon GO". During November - December 2017 this search then yielded 5880 results, of which most papers had no empirical research approach. The key papers from this part of the study are referred to in this paper. They stand out methodologically and address the social aspects of gameplay. The literature was later April-June 2019 revisited after the analysis of collected images to update knowledge on research conducted 2018 and 2019. The research process is outlined in the following figure 1.

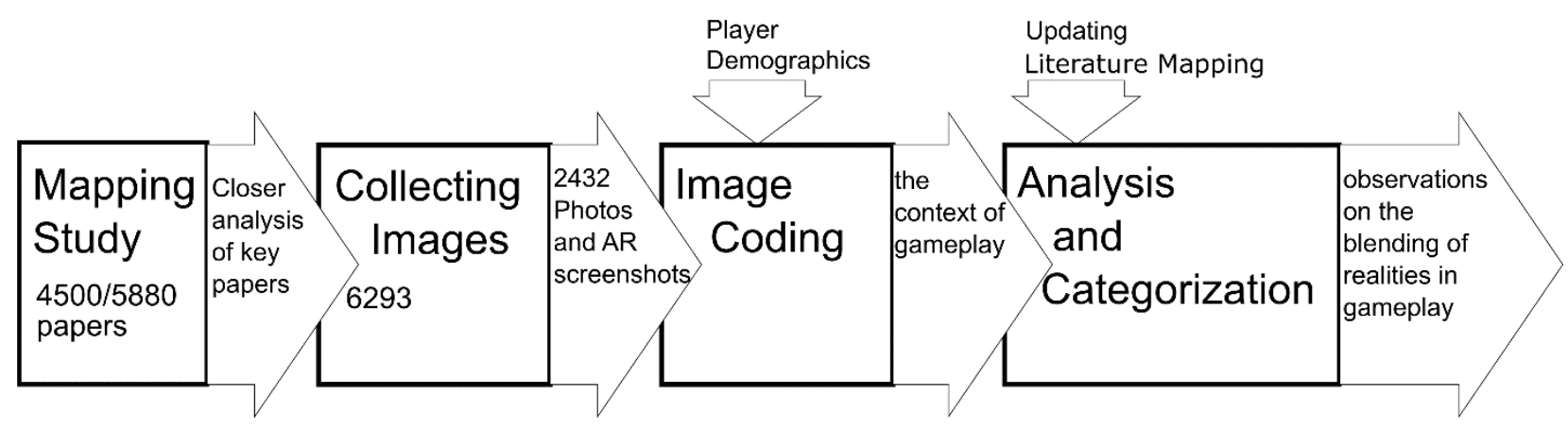

Figure 1. Process of this research.

The scope of this research spans through eight cities across five Nordic countries, including Finland (Helsinki, Tampere, Turku, and Oulu), Sweden (Stockholm), Denmark (Copenhagen), Norway (Oslo) and Iceland (Reykjavik). The collection of images was conducted during November 16 to December 22 in 2017. The sample contained images posted on social media sites 
since the release of Pokémon GO, July 2016. The search phrases used on Instagram were: \#pokemongo suffixed with the name of a particular city, both in English and the native language. In Twitter it was possible to use Boolean search with phrases \#pokemongo and the name of the city as separate. Although images from three social media sites were analyzed, the main contributor for the analysis was Facebook, with $65,3 \%$ of the analyzed photos. In Facebook, the observers signed up for Pokémon GO player groups for each city (Pokémon GO Island for images from Reykjavik).

The Facebook groups included had 34081 members and active discussion at the time of this study. 712 (51\% Male and 49\% Female) individual people submitted the images that were part of the sample from Facebook. Demographics data was retrieved only based on Facebook, it is however safe to say that although some players post AR snapshots and photographs more often than others, the analyzed images represent many and form an adequate sample size of the whole population of Pokémon GO players.

The total number of images reviewed was 6293, of which the screenshots of the Pokémon GO user interface were excluded. They contributed to $61.4 \%$ of the images in social media communications of the players. Photos, either AR snapshots (13.2\%) or other photos $(21.2 \%)$ were selected for closer inspection. In the end there were 2432 images left for analysis. The topical themes in the images are much as described in a prior study (Majgaard \& Larsen, 2017). Since the images analyzed are not only on public, but some are in semi-public forums, it was found necessary to do the coding amongst the researchers and therefore no material was handed to third parties. Furthermore, the discussion between the two observers during the process of analysis was valuable to the outcome of this research. 
The coding schema is based on a schema from Selfiecity, for studying the urban context of selfies in social media (Tifentale \& Manovich. 2015). The schema was adjusted after preliminary side by side coding of roughly 400 images, as parameters for image tagging e.g. coding, relevant for the objective of the study were needed. The final coding schema (Table 1) contains two LBMG specific categories on blending of hybrid realities (Blending) and blending of daily life and gameplay. The activity category (from the original schema) provides a detailed view on what kind of daily activities are blended with gameplay. Arts and crafts specific code, "Arts and crafts" was added as there are noticeable number of photos related crafts and fan art among the full image collection. These images include indirect screenshots, for example, the photos that are taken to present a screenshot or image-collages. Since many of the analyzed images do not contain game elements especially in the photo category, we also added relevant codes for these, such as "Undefined" and "No Blending". These images are scoped in such manner that it isimpossible to code them in a way relevant to the category. 
Table 1. The coding schema for first stage of analysis and categorization of the image topics

\begin{tabular}{|c|c|}
\hline Category & Code \\
\hline Subject* & $\begin{array}{l}\text { Person, Object, Food, Group, Public event, Landscape, } \\
\text { Advertisement, Screenshot Architecture, Interior, Pet, Selfie, } \\
\text { Edited image }\end{array}$ \\
\hline AR view & Photo, AR view \\
\hline Space & Indoor, Outdoor \\
\hline Indoors & Private, Public \\
\hline Outdoors & Built, Urban nature, Nature \\
\hline Use situation & Playing, Daily Play, Undefined \\
\hline Blending of realities & $\begin{array}{l}\text { Digital to Physical, Both ways between digital and physical, } \\
\text { Physical to Digital through Items, Digital to physical through } \\
\text { Imagination, Undefined }\end{array}$ \\
\hline Activity & $\begin{array}{l}\text { Income, Nutrition, Mobility, Education, Social, } \\
\text { Consumption, Caretaking, Personal care, Arts and crafts, } \\
\text { Leisure }\end{array}$ \\
\hline
\end{tabular}

*Coding of the AR view was defined by the background (as they would have otherwise always been considered mere screenshots)

Initial coding was done using a software called ImageTagger (SPIN Unit, 2015). Two observers conducted the coding. Agreement testing was conducted using point-by point agreement by 
Cohen's Kappa (Bakeman \& Quera, 2011; Fleiss, Levin \& Paik, 2013) with a sample of 114 photos. On image subject the observers according to the guidelines from Altman (1999) had good agreement $(\kappa=0.607)$, in recognizing AR view in images very good agreement $(\kappa=0.965)$, on indoor and outdoor spaces and all their subcategories the agreement was moderate $(\kappa=0.505)$, as it was on observing blending of daily life and gameplay $(\kappa=0.569)$, on blending of realities $(\kappa=0.581)$ and on interpreting activities from the images $(\kappa=0.588)$. All values had $\mathrm{p}<0.001$. The subsequent analysis and categorization followed general qualitative coding principles (Charmaz 2011). The identified topics were used for categorizing the data until no new themes arose from the material.

\section{Results}

The main subject in the images varied (Figure 2). There are more landscapes in images taken using AR view (21.7\%), possibly because the individual pokémon are often at the focal point of those images and outdoor landscapes offer a suitable backdrop for them. Mere photographs in turn depict objects (19.9\%), such as game related purchases. In AR screenshots the main topic was an object only in very few images (2.7\%). These differences warrant a reason to keep the analysis of the two differing types of images separate.

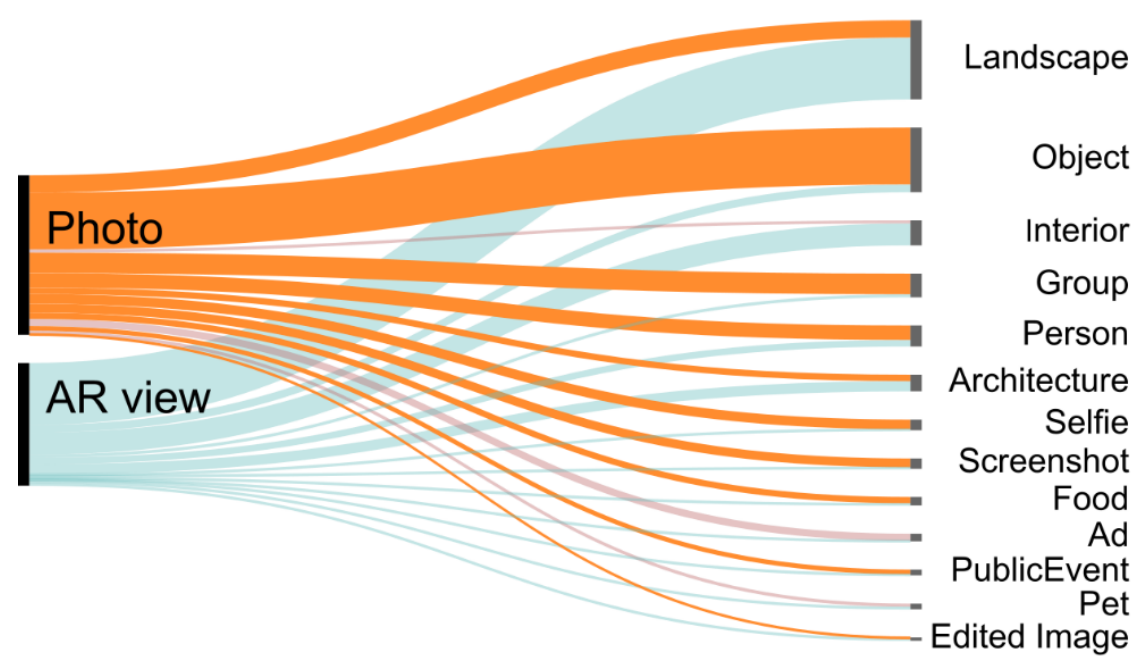


Figure 2. The main subjects in analyzed images. The alluvial diagram is structured so that thickness of each line is proportional to the number of images coded.

Tifentale \& Manovich (2015) notice in their study that the number of selfies among city specific images posted in social media sites is quite low 3-5\%. In Pokémon GO communities, the percentage is $3.7 \%$. This also complements the knowledge that the games and gameplay are usually the topic of content in game related forums (Griffiths et al., 2016; Al-Rawi \& Consalvo, 2019).

\subsection{Context of gameplay}

The most dominant environment for play is urban build environments (37.6\%) (Figure 3). Urban green areas $(20.0 \%)$ are second and only a few photos from cemeteries can be observed. Indoor gameplay $(19.6 \%)$ in private $(11.4 \%)$ or public places $(8.2 \%)$ is frequent. Although it does not match outdoor gameplay in popularity it contributes to one thirds of gameplay depicted in the images.

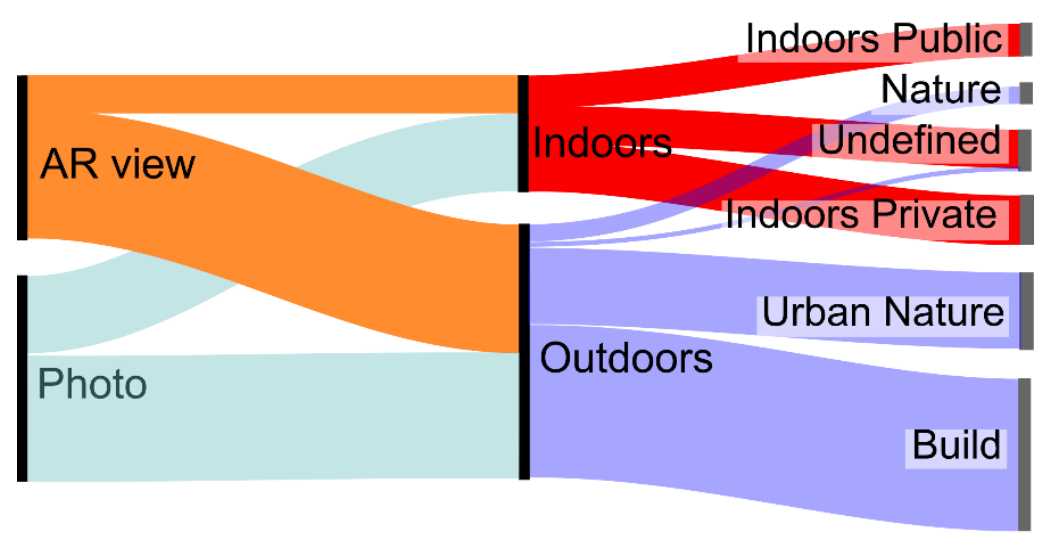

Figure 3. A graph on how context of gameplay is divided on 2189 images where the context was observable. The alluvial diagram is structured so that thickness of each line is proportional to the number of images coded. 
Indoors people seem to prefer to play both in public and private locations. The public locations are cafes or shops in between routes through outdoor spaces and the private locations are usually homes. The gameplay indoors is not as intense as outdoors gameplay and it is more entwined with daily life activities (Figure 4). In AR images taken outdoors one can see mostly landscapes in private indoors locations the depicted subjects show more variability. Build urban outdoors environment is the most common context for playing the game, despite the players often group in urban green areas.
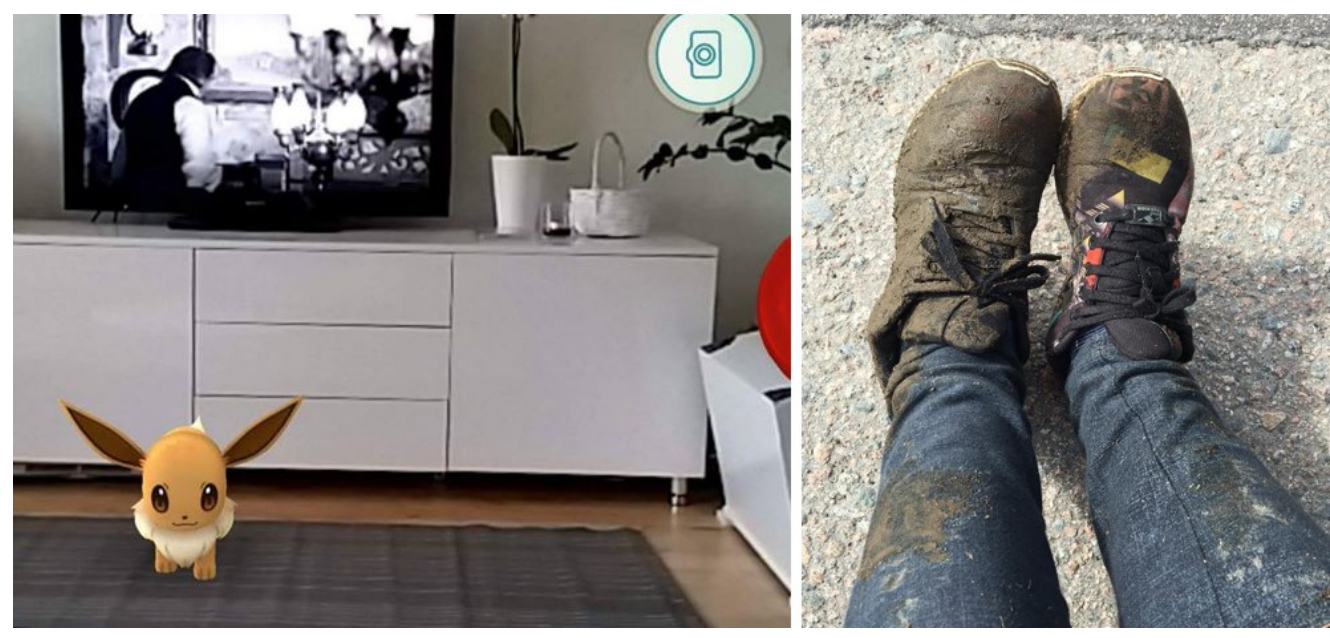

Figure 4. Intensity of gameplay indoors and outdoors, (left) a player states that they have a new low maintenance pet that only wants for you to throw a ball at it from the sofa once in a while and (right) a player comments her muddy feet by wondering what went into her while chasing a pokémon into the woods. Figures with permission from Mia Niittyaro-Nygren (left) and the copyright holder wishes to stay anonymous (right).

\subsection{Use situation: Blending of daily life and gameplay}


Many of the activities such as Income $(0.4 \%)$ and Education $(0.4 \%)$ are underrepresented in the gathered material and Leisure (56.4\%) is obviously overrepresented as gameplay even when blended with daily life can still be observed as leisure activity. The rules of online communities often require the communication to be game related. Fairly few such images where context of the activities of education or income are blended with gameplay were identified. This suggests that work and school are still somewhat nonplayful contexts when it comes to LBMGs. It is also possible that people are careful not to report playing while working especially if the photos are shared on social media (Figure 5).

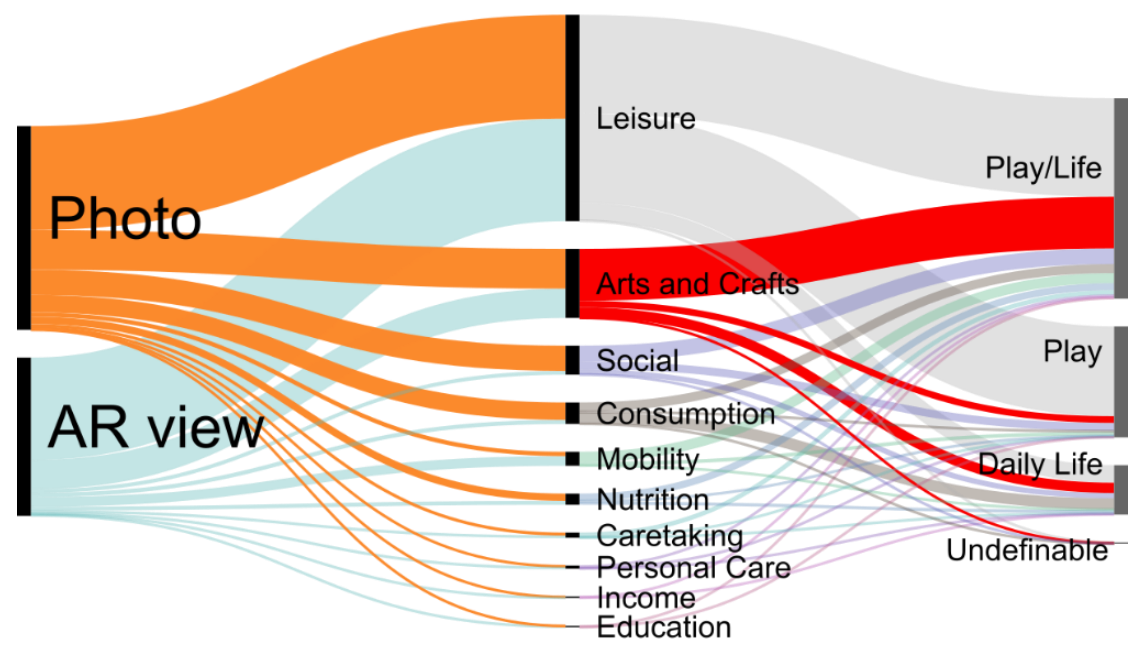

Figure 5. The activities presented in 2328 images where the activity was perceivable or deductible. The activities are in the middle node and on the right node lies the Use situation e.g. observed blending of daily life and gameplay (there were 8 undefinable images in this category).

Players capture pokémon while having lunch, walking their dog, commuting, or shopping for groceries (Figure 6). These activities are perceived as daily life activities. Social activities are high in the most popular activity to blend with gameplay, but the social activity in analyzed images is mostly taking part in player gatherings, hence the activity would not exist without the game. 

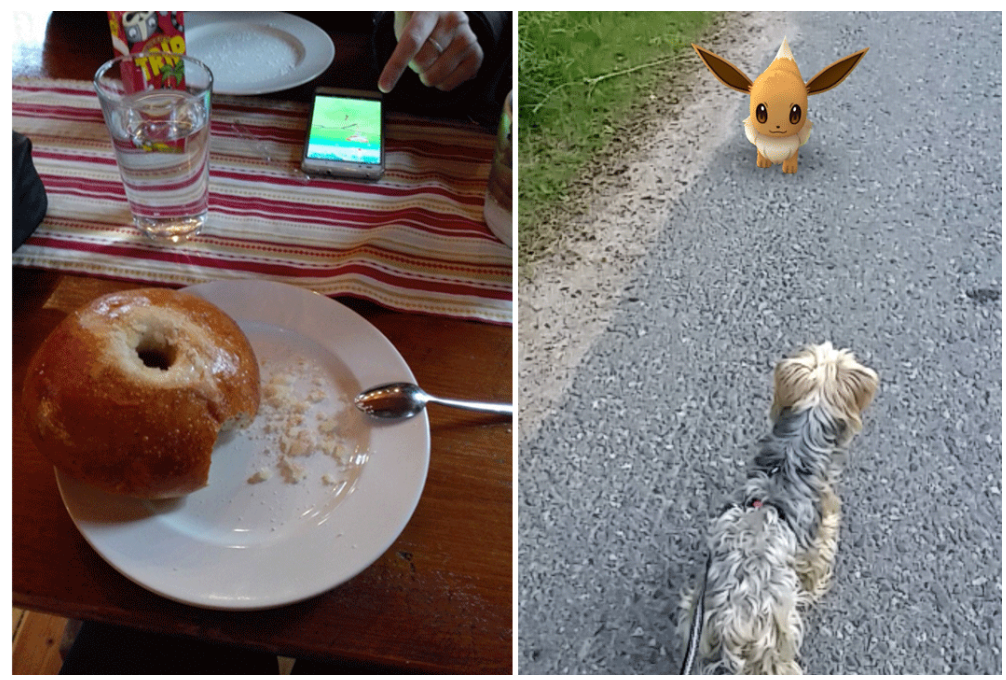

Figure 6. Typical activities, (left) having a snack with a friend and playing, coded as Nutrition (right) and walking a dog while playing, which was coded as Caretaking. Figures with permission Rebeca Dragomir (left) and Anu Vehkomäki (right).

Arts and Crafts (18.7\%) was the most common clearly defined activity observed in the images. Fan art of such a popular game can be expected [27]. The players go as far as placing their sculptures on a street walk and capturing a photo of it as if it were AR view from the game (Figure 1). The extend and variety of the player creations tells how the game narrative is influencing daily choices of the players (Figure 7). 


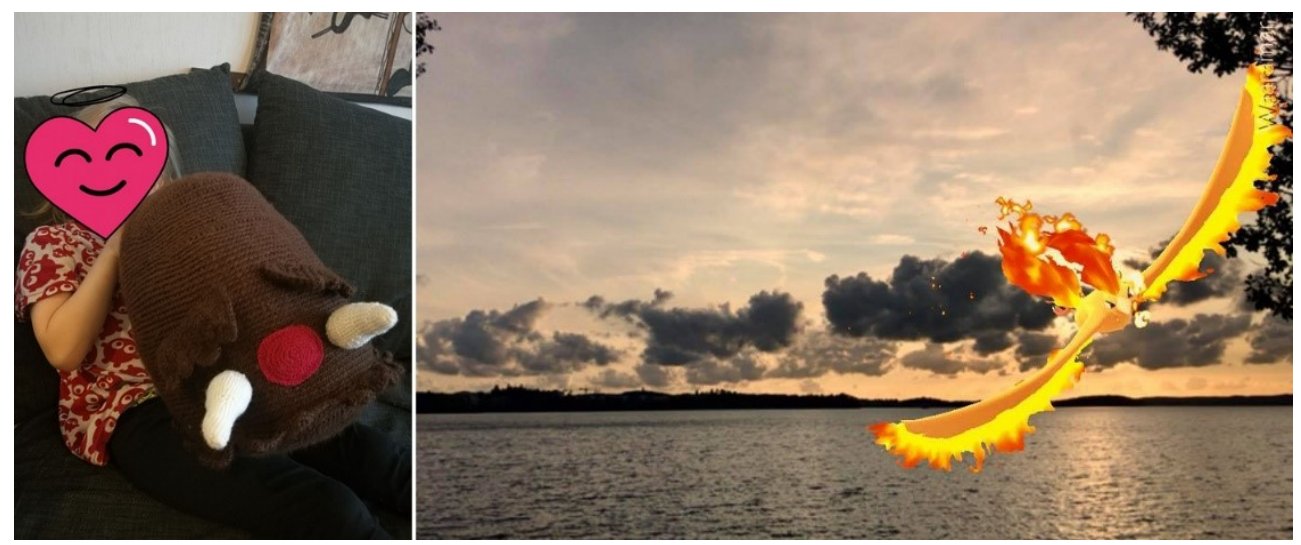

Figure 7. Fanart created by the players, knitting and embedding the pokémon to a physical environment (left).A rare pokémon soaring through the sky (right). Figures with permission from Kaisa Soukka (left) and Markus Sebastian Waara (right).

There is also a specific form of fanart where the digital game and physical landscapes or objects are used to create more serious pieces of art (Figure 7, right). Some players specialize in "nature photography" of rare pokémon.

\subsection{Combining realities}

In how blending of realities is depicted in the analyzed images the AR images and photos differ the most. Digital overlay of the AR view creates an obvious blending in the direction from digital to physical environment (Figure 8) and this blending is most visible in AR snapshots. The No Blending images were such that there was no observable blending between the digital or other contents of the game and physical environment detectable in those images. Most of these were landscape photos. 


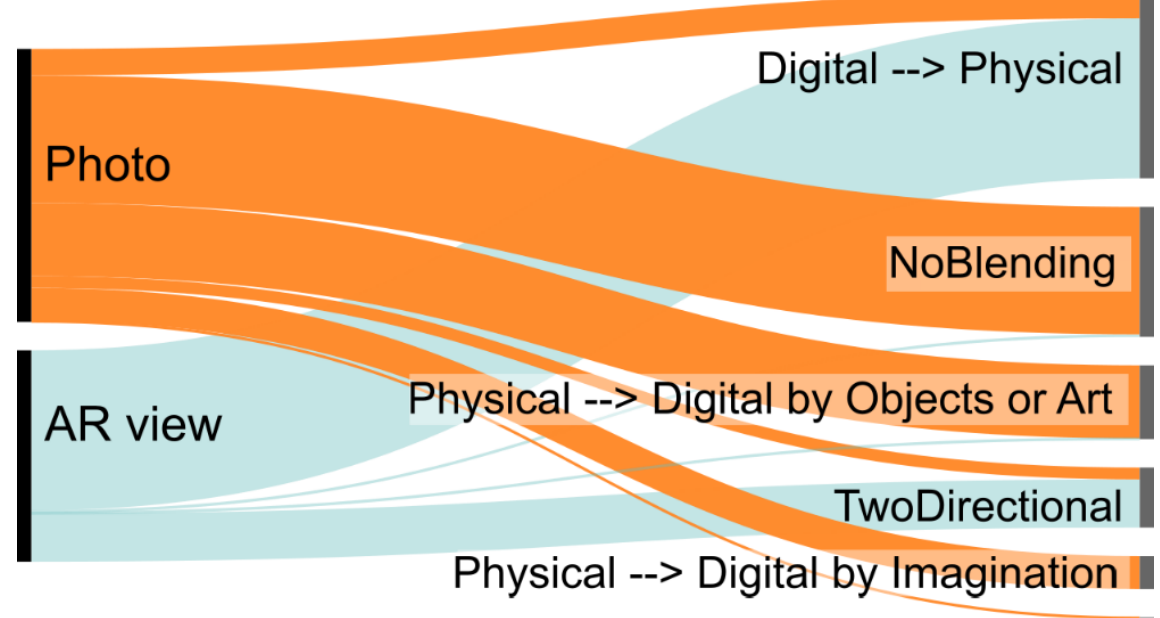

Figure 8. In what frequencies photos and AR screenshots show blending between digital gamespace and the physical world.

Furthermore, two directional blending of realities, where the digital contents is projected against a physical backdrop in a way that the physical environment affects how the digital is situated is made possible by the AR view of the game (Figure 9.), although some two-directional blending can be observed from some photos too. AR screenshots that show pokémon embedded in the daily activities of the players with creativity and timing (Figure 9.) are more commonplace than mere chance would allow. 

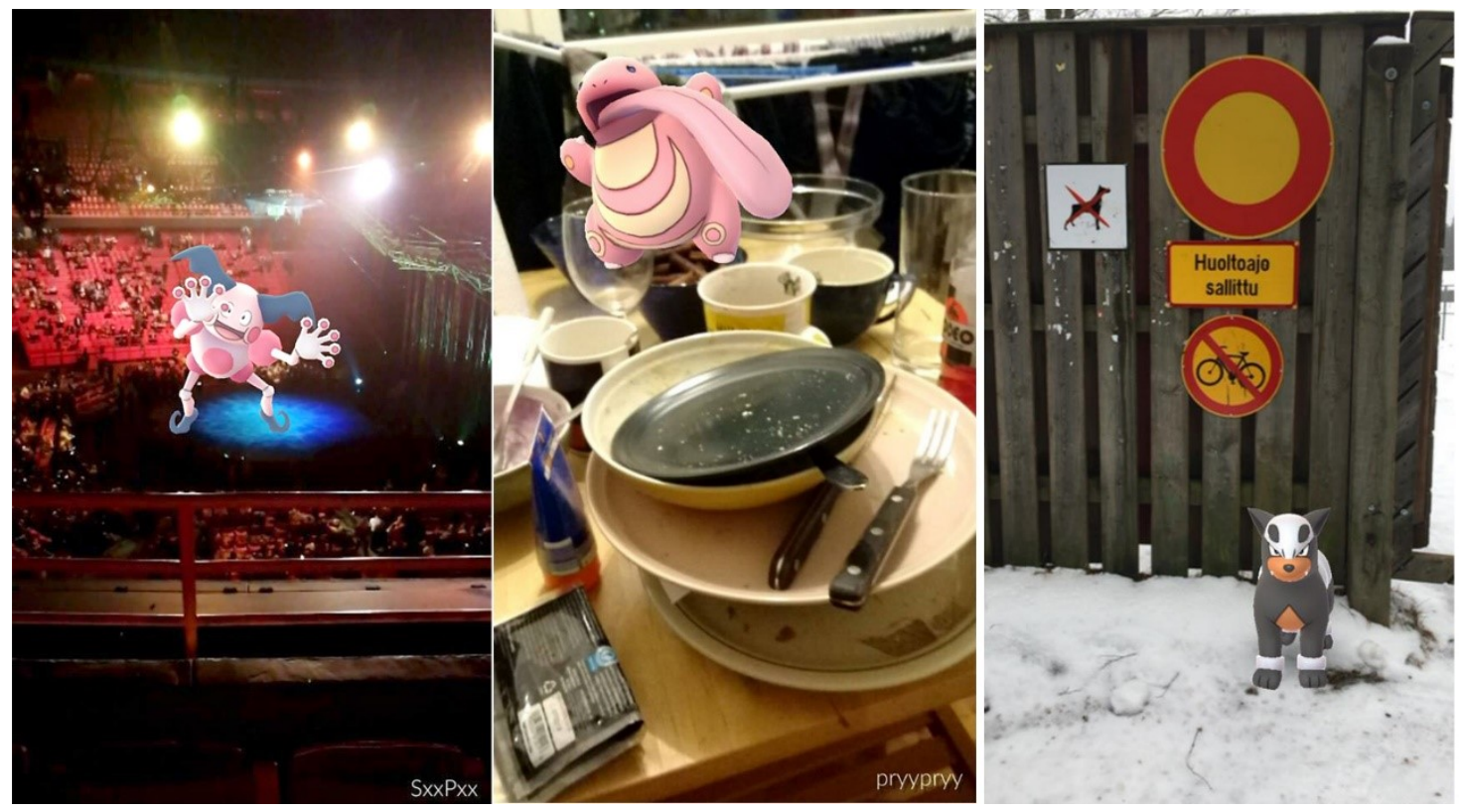

Figure 9. Two way blending of realities (also daily life and gameplay indoors), (left) playing Pokémon GO in AR mode at a public location while attending Cirque du Soleil performance in Stockholm, (middle) an indoors private location a player is having Lickitung do his dishes. Embedding digital pokémon into context of daily life (right) in a way that further shows two-way influence between digital and physical environments through composition and timing. Figures with permission from Jean G. Stephan (left), Pyry Ekholm (center) and Christian Seppänen (right).

The formation of schemas and the blending effect from physical to virtual through imagination can be observed in images where physical world objects are perceived as pokémon (Figure 10). 


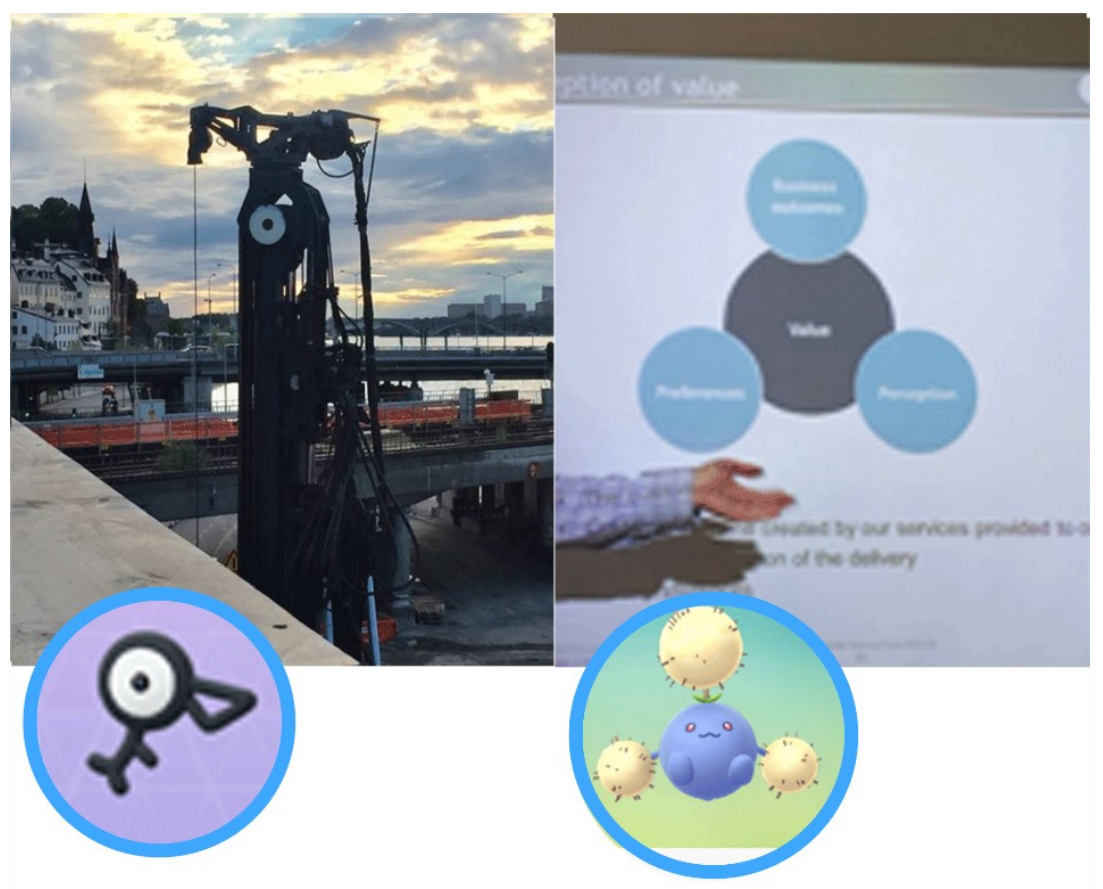

Figure 10. Pokémon becoming a part of mental schemas of players and blending of realities through imagination, the pokémon the features in the environment are compared to below. This phenomenon is also known as called game transfer (Ortiz de Gortari, 2018). Figures with permission from an anonymous player form the Facebook group Pokémon GO Stockholm (left) and an anonymous player (right).

\section{Discussion}

Blending of realities and the blurry boundary between daily life and gameplay is made observable in the images the players post in social media sites. In this article photos of gameplay and AR screenshots revealed new qualities of this blending and how it is structured. Daily life and gameplay proved to be so deeply interlaced that unblurring their blendingcompletely does not seem possible. Regardless of how little use the AR feature has in gameplay, and whether it upkeeps player retention (Alha et al., 2019), the AR screenshots and other photos posted in social media 
sites are a specific form of communication for the players. Amongst the images the players use to communicate in social media, there are plenty of AR images although they do not dominate. In fact, most of the images are screenshots from the game user interface such as images from hatching eggs (pokémon eggs require a 2-10km walk to hatch). A typical comment for an image depicting outdoors scenery is: "On a Sunday walk through the rain and $100 \%$ raikoun" or "Spotted this while pokehunting". Although the presented analysis is mainly based on visual ethnography, observations are affected by the short commentary the players submitted alongside their images.

\subsection{Playful urban space, where?}

Homo ludens, what Huizinga (1955) calls modern human, is intrinsically playful, and that playfulness is passed on to the spaces we occupy or observe (Sicart, 2014, 2016; de Souza e Silva., 2009). Some locations and activities for Pokémon GO play are more playful than others. In LBMGs, the players are in motion and playful urban space could be perceived to be towards the direction of the smallest resistance. The players roam on the city streets and in their homes, e.g. the areas they have access to. The resistance though may be in the form of physical walls and obstacles but also in the form of common norms. Despite the post-launch news on Pokémon GO players mindlessly wandering at locations where discretion is required, this research shows that players roam in commonly accessible places. Build urban environment is in fact the most common context for gameplay. Personal privacy does not form such obstacle though, the playful urban space includes the players' homes, beds, and bathrooms. However, they do not often play in the office or at school. This finding suggests that the entwined gameplay is in the daily lives of the players. In contrast, professional life is rarely blended with leisure. These observations support to the notion of distinguishable magic circle (Huizinga, 1955; Stenros, 2012) in LBMGs. Magic circle being the boundary between gameplay and daily life (Huizinga, 1955), of which's 
distinguishability in pervasive and therefore also LBMGs, has been debated (Montola, 20015; Stenros, 2012).

In Pokémon GO the observable activities and the crowds are often concentrated around locations called Gyms, Pokestops, or the spawn locations of the Pokémon, which are all situated outdoors or public indoor spaces (Ishii et al. 2011; Wang et al. 2018). Players also tend to explore more outside their usual daily routes (Evans \& Saker, 2018). However, these game specific locations can be accessed from indoors when they are close enough, and pokémon can be lured to one's person using a digital item called incense. So, the game is and can be played indoors. This aspect is somewhat neglected in current literature of the game and the design implications of the indoor play could be taken into better consideration. The possibility to play the game indoors, especially at one's home offers different lower level of intensity of gameplay and a more intimate blending of daily life and gameplay.

\subsection{Blending realities}

It is notable that the AR imagery shows how the social media posts of photos depict the two-way influence between the physical world and the virtual one. People pose alongside the pokémon and appear to hold them or take snapshots of the digital creatures sitting on the edge of a wineglass or a toilet seat. The social media sites can be interpreted as one observed layer in the hybrid space (Alavesa, 2018; de Souza e Silva., 2009). The digital game contents and the physical context for gameplay present other realities. There is a clear interplay between the digital and physical in the analyzed images. In addition, there is interplay between the minds and imaginations of the players towards both digital and physical realms. The phenomenon of association between physical and digital stimuli while playing a game is called game transfer (Ortiz de Gotari, 2018). The players can imagine pokémon are present in the physical world or have visual and auditory hallucination 
like experiences while playing the game. This phenomenon has been studied in location-based gaming with AR, such as Pokémon GO and Ingress, where constant shifting of attention i.e. cognitive jumps between digital and physical environments is required in gameplay (Ortiz de Gotari, 2018; Sifonis, 2016). Recent studies also show physiological adaptations in Pokémon GO as restructuring of areas in areas of brain responsible for visual processing (Janini \& Konkle, 2019). The game transfer observed in this study is multidirectional; hand-crafted pokémon are situated into the physical streets where digital pokémon would appear, every-day objects are seen resembling pokémon regardless of context, and selfies or portraits are taken posing with digital creatures tied to their location by geocoordinates and AR targeting. These phenomena show that minds and imaginations tie the physical and digital in similar manor as physical objects (Spallazzo \& Mariani, 2018) or locationing (de Souza e Silva \& Sutko, 2018). Sociability shown by the images is not that apparent, although many images were in the end reported in the social context, in social media.

\subsection{Additional observations and outlier behavior}

It is possible to see Pikachu wearing a red cap, a gnome hat, in pictures taken during the holidays. This kind of two directional temporal blending shows that there already is some degree of blending of realities by design in Pokémon GO. Other examples of this are water type pokémon spawning closer to a body of water, or pokémon who cannot fly being tracked on a surface and flying pokémon hovering in the air with a hint of a shadow beneath. In this study, these forms of blending were interpreted one-directional from digital to physical.

At some point there has been a meme where people wait for a specific pokémon to spawn (either Krabby or Hypno) and run to the nearest toilet to take an AR snapshot. The images stand out from the sample, but they are not that frequent. They also show the how willing the players are to publish 
images from private environment to social media. The private setting does not however mean that the images are intimately private as there are hardly ever people in the images, just pokémon. Considering that Pokémon GO can also be characterized as an exergame, it is surprising that there are only few images that reflected this aspect of gameplay (an image of a smartwatch that instead of a pedometer showed when an egg would hatch).The game does make people exercise and move in a subtle way which may be why people do not self-report (using imagery) of this aspect of gameplay. Many images of worn out shoes were shared.

\subsection{Limitations and future work}

This article aims at providing a view to the boundary between daily life and gameplay, with the aim of removing some of the blurriness, yet in PokémonGO the daily life and gameplay become so intermingled that they are inseparable. While our coding schema was structured so that the codes were discrete, this does not remove the ambiguity of reality the analyzed images present. This also shows in the low agreement scores between observers. In other words, the ways and depth of blending between daily life and gameplay observed in this study form a continual spectrum. Thecodes and categories usedfor analysis only present a rough sampling from that

spectrum. A closer sampling and inspection of the blending between daily life and gameplay could reveal more about this blending, however based on this research it can be easily concluded that the blending is very noticeable.

In this study the observers played actively only in three of the cities in the sample of the study. However, they are familiar with the Nordic urban landscape to the extent that they could assess the context of gameplay. Especially making the distinction between private and public interior space is easier when one is familiar with the public interior spaces depicted. The ambiguity of the 
observer phenomenon and interpretability of the results contributed to the low agreement scores between the two observers.

This study presents a Nordic perspective on LBMGs and Pokémon GO is a global phenomenon, which is known to reflect into how the gameplay is communicated via social media (Al-Rawi \& Consalvo, 2019). Expanding the analysis to material for social media sites globally would make it possible to create a global map on playful spaces, which would be an interesting target for future research.

The earliest images analyzed by us were submitted within a week from the global release of the game, hence there is a retrospective nature in this study. People are however known to curate and edit their images in social media sites (Hogan \& Quan-Haase, 2010), hence there may be a slight bias towards more recent photos in the analyzed images, which is on the other hand complemented by the slope in popularity of the game.

In game related forums and communities, the game itself frames the discussion. Gameplay related sites do not follow the same content structure as other social media communication (Hu et al. 2014). Some players are very prolific fan artists and frequently submit AR images with personal watermarks. Such participants were tracked to their personal social media sites to check that, the sites do not contain Pokémon images or images of gameplay, but personal images of life and work. Therefore, the AR images or photos analyzed do not reveal much of the personality of the players. On the other hand, this suggests that the images are reliably on topic: Pokémon GO related events and gameplay.

Many recent updates, research quests, surprise encounters and making the AR camera (GO Snapshot) available through pokémon inventory, have increased the use of AR feature in the game and this has resulted into an influx of game related AR images in the social media groups. This 
study was however conducted during a time when the use of AR was not as forced by game mechanics and this gives this research a unique perspective. Many of the images taken and analyzed for this study have required timing, luck and sometimes patience from the players.

\section{Conclusion}

Whether the blending of realities in LBMGs is called cyber-physical symbiotic social networks, or hybrid information spaces, games such as Pokémon GO do blend digital and physical in addition to blending the daily life and gameplay. This paper addresses the gameplay of Pokémon GO and shows how the players share their gaming experience through pictures that are depicting the blending of different realities. Findings of this study demonstrate that digital and visual ethnographic approach can provide rich insights about the context of the gameplay. In this study some of the boundaries of the playful urban space and activities were identified. Norms do indeed restrict the context of gameplay and how closely daily life and play are entwined. It was also observed that indoor gameplay is commonplace, and the players also play in their beds and bathrooms frequently. To the game designers and developers it can be proposed to go beyond the conventional wisdom of outdoor-focused location-based mobile game design and pay attention to the gameplay behaviors in different contexts (indoor-outdoor, public-private), which can lead to more creative game design and enriched game experience.

\subsection{Acknowledgments}

First author has received funding from Business Finland funded project Reboot Finland IoT Factory 33/31/2018 and PRINCE (81536), supported by Academy of Finland 6Genesis Flagship (318927). This research has been conducted following the ethical requirements established by 
Finnish advisory board on research integrity. The gathered material has been handled and informed consent from the participants was obtained in accordance with Finnish laws. Permission from the original copyright holders was obtained for publishing the images in this paper.

\subsection{Disclosure statement}

The Authors declare that there is no conflict of interest with respect to the authoring and publication of this article.

\section{References}

Al-Rawi, A., \& Consalvo, M. (2019). Video Game Engagement on Social Media in the Middle East. In Video Games and the Global South (pp. 234-252). Pittsburgh, PA, US: ETC Press.

Alavesa, P. (2018). Playful appropriations of hybrid space: combining virtual and physical environments in urban pervasive games. University of Oulu, Oulu, Finland. Retrieved from http://urn.fi/urn:isbn:9789526221380

Alha, K., Koskinen, E., Paavilainen, J., \& Hamari, J. (2019). Why do people play location-based augmented reality games: A study on Pokémon GO. Computers in Human Behavior, 93, 114 122.

Althoff, T., Jindal, P., \& Leskovec, J. (2017). Online actions with offline impact: How online social networks influence online and offline user behavior. In Proceedings of the Tenth ACM International Conference on Web Search and Data Mining (pp. 537-546). ACM. https://doi.org/10.1145/3018661.3018672

Althoff, T., White, R. W., \& Horvitz, E. (2016). Influence of Pokémon Go on Physical Activity: Study and Implications. Journal of Medical Internet Research, 18(12), e315. https://doi.org/10.2196/jmir.6759

Altman, D. G. (1990). Practical statistics for medical research. CRC press. 
Androutsopoulos, J. (2008). Potentials and limitations of discourse-centred online ethnography. Language@Internet, 5(8).

Azuma, R. T. (1997). A survey of augmented reality. Presence: Teleoperators and Virtual Environments, 6(4), 355-385. https://doi.org/10.1162/pres.1997.6.4.355

Bakeman, R., \& Quera, V. (2011). Sequential analysis and observational methods for the behavioral sciences. Cambridge University Press.

Baum, F. (1995). Researching public health: behind the qualitative-quantitative methodological debate. Social Science \& Medicine, 40(4), 459-468. https://doi.org/10.1016/02779536(94)E0103-Y

Beaulieu, A. (2004). Mediating ethnography: objectivity and the making of ethnographies of the internet. $\quad$ Social Epistemology, $\quad 18(2-3), \quad 139-163$. https://doi.org/10.1080/0269172042000249264

Benford, S., \& Giannachi, G. (2011). Performing Mixed Reality. The MIT Press.

Brulliard, K. (2016, July 13). If you must play Pokémon Go, 'catch' some real animals while you're at it. Washington Post. Retrieved from https://www.washingtonpost.com/news/animalia/wp/2016/07/13/if-you-must-playpokemon-go-catch-some-real-animals-while-youre-at-it/

Charmaz, K. (2019). Handbook of Interview Research. In pages 675-694. SAGE Publications, Inc. https://doi.org/10.4135/9781412973588

Colley, A., Thebault-Spieker, J., Lin, A. Y., Degraen, D., Fischman, B., Häkkilä, J., ... Wenig, N. (2017). The geography of Pokémon GO: beneficial and problematic effects on places and movement. 1179-1192. ACM. 
Dorward, L. J., Mittermeier, J. C., Sandbrook, C., \& Spooner, F. (2017). Pokémon go: benefits, costs, and lessons for the conservation movement. Conservation Letters, 10(1), 160-165. https://doi.org/10.1111/conl.12326

Evans, L., \& Saker, M. (2018). The playeur and Pokémon Go: Examining the effects of locative play on spatiality and sociability. Mobile Media \& Communication, 2050157918798866.

Fleiss, J. L., Levin, B., \& Paik, M. C. (2013). Statistical methods for rates and proportions. John Wiley \& Sons.

Griffiths, M., Lewis, A., de Gortari, A., \& Kuss, D. (2016). Online forums and solicited blogs: innovative methodologies for online gaming data collection. Studia Psychologica, 15(2), 101122.

Hine, C. (2000). Virtual ethnography. Sage Publications Ltd.

Hogan, B., \& Quan-Haase, A. (2010). Persistence and change in social media. Bulletin of Science, Technology \& Society, 30(5), 309-315. https://doi.org/10.1177/0270467610380012

Hu, Y., Manikonda, L., \& Kambhampati, S. (2014). What We Instagram: A First Analysis of Instagram Photo Content and User Types. In Icwsm.

Huizinga, J. (1955). Homo Ludens: A Study of the Play Element in Culture. London, United Kingdom: Routledge \& Kegan Paul.

Ishii, A., Ajito, M. \& Kawahata, Y. (2016). Analysis of Pokémon GO using sociophysics approach. 2016 IEEE International Conference on Big Data (Big Data), 3986-3988. https://doi.org/10.1109/BigData.2016.7841084

Janini, D., \& Konkle, T. (2019). A Pokémon-sized window into the human brain. Nature Human Behaviour, 3(6), 552-553. https://doi.org/10.1038/s41562-019-0594-6

Kozinets, R. V. (2015). Netnography. Wiley Online Library. 
Lee, J. H., Windleharth, T., Yip, J., \& Schmalz, M. (2017). Impact of location-based augmented reality games on people's information behavior: A case study of Pokémon Go. IConference 2017 Proceedings. https://doi.org/10.9776/17218

Liu, L., Wagner, C., \& Suh, A. (2017). Understanding the Success of Pokémon Go: Impact of Immersion on Players' Continuance Intention. In D. D. Schmorrow \& C. M. Fidopiastis (Eds.), Augmented Cognition. Enhancing Cognition and Behavior in Complex Human Environments: 11th International Conference, AC 2017, Held as Part of HCI International 2017, Vancouver, BC, Canada, July 9-14, 2017, Proceedings, Part II (pp. 514-523). Cham: Springer International Publishing. Retrieved from https://doi.org/10.1007/978-3-319-58625$0 \_37$

Lotay, A. (2016). Disclosure of psychological distress by university students on an anonymous social media application: an online ethnographic study.

Lynch, K. (1960). The image of the city (Vol. 11). MIT press.

Madden, R. (2014). Animals and the Limits of Ethnography. Anthrozoös, 27(2), 279-293.

Majgaard, G., \& Larsen, L. J. (2017). Pokémon GO: A Pervasive Game and Learning Community. In ECGBL 2017 11th European Conference on Game-Based Learning (p. 402).

Manifold, M. C. (2009). Fanart as craft and the creation of culture. International Journal of Education Through Art, 5(1), 7-21. https://doi.org/10.1386/eta.5.1.7_1

Markham, A. N., \& Baym, N. K. (2008). Internet inquiry: Conversations about method. Sage.

Montola, M. (2005). Exploring the edge of the magic circle: Defining pervasive games. In Proceedings of DAC (Vol. 1966, p. 103).

Montola, M., Stenros, J., \& Waern, A. (2009). Pervasive games: theory and design. Morgan Kaufmann Publishers Inc. 
Niantic. (2017). Pokémon GO (Version 0.69.1) [Android 4.4 and up]. Niantic, Inc. Retrieved from https://play.google.com/store/apps/details?id=com.nianticlabs.pokemongo

O'Hara, K., Glancy, M., \& Robertshaw, S. (2008). Understanding collective play in an urban screen game. Proceedings of the 2008 ACM Conference on Computer Supported Cooperative Work, 67-76. San Diego, CA, USA: ACM.

Ortiz de Gortari, A. B. (2018). Empirical study on Game Transfer Phenomena in a location-based augmented reality game. Telematics and Informatics, 35(2), 382-396. https://doi.org/10.1016/j.tele.2017.12.015

Paavilainen, J., Korhonen, H., Alha, K., Stenros, J., Koskinen, E., \& Mayra, F. (2017). The Pokemon GO Experience: A Location-Based Augmented Reality Mobile Game Goes Mainstream. In Proceedings of the 2017 CHI Conference on Human Factors in Computing Systems (pp. 2493-2498). Denver, Colorado, USA: ACM. https://doi.org/10.1145/3025453.3025871

Paasovaara, S., Jarusriboonchai, P., \& Olsson, T. (2017). Understanding collocated social interaction between Pokémon GO players (pp. 151-163). Presented at the Proceedings of the 16th International Conference on Mobile and Ubiquitous Multimedia, ACM. https://doi.org/10.1145/3152832.3152854

Pettigrew, K. E. (1999). Waiting for chiropody: contextual results from an ethnographic study of the information behaviour among attendees at community clinics. Information Processing \& Management, 35(6), 801-817. https://doi.org/10.1016/S0306-4573(99)00027-8

Pittman, M., \& Reich, B. (2016). Social media and loneliness: Why an Instagram picture may be worth more than a thousand Twitter words. Computers in Human Behavior, 62, 155-167. https://doi.org/10.1016/j.chb.2016.03.084 
Relph, E. (1976). Place and placelessness (Vol. 1). Pion.

Schwartz, D. (1989). Visual ethnography: Using photography in qualitative research. Qualitative Sociology, 12(2), 119-154. https://doi.org/10.1007/BF00988995

Senior, K. A., \& Chenhall, R. D. (2012). Boyfriends, babies and basketball: Present lives and future aspirations of young women in a remote Australian Aboriginal community. Journal of Youth Studies, 15(3), 369-388. https://doi.org/10.1080/13676261.2012.663890

Sifonis, C. (2016). Game transfer phenomena in the augmented reality game, Ingress. International Academic Conference on Meaningful Play, Michigan, United States of America.

Sicart, M. (2016). Play and the city. Navigationen-Zeitschrift Für Medien-Und Kulturwissenschaften, 16(1), 25-40.

Sicart, M. (2014). Play matters. MIT Press.

Skågeby, J. (2008). Gifting technologies: Ethnographic studies of end-users and social media sharing. Department of Computer and Information Science, Linköpings universitet.

Southerton, C. (2013). Zombies, run!': Rethinking immersion in light of nontraditional gaming contexts. Transmedia: Storytelling and Beyond Digital Interfaces.

de Souza e Silva, A. (2016). Pokémon Go as an HRG: Mobility, sociability, and surveillance in hybrid spaces. Mobile Media \& Communication, 5(1), 20-13. https://doi.org/10.1177/2050157916676232

de Souza e Silva, A. (2009). Hybrid Reality and Location-Based Gaming: Redefining Mobility and Game Spaces in Urban Environments. Simulation \& Gaming, 40(3), 404-424. https://doi.org/10.1177/1046878108314643 
de Souza e Silva, A., \& Sutko, D. M. (2008). Playing Life and Living Play: How Hybrid Reality Games Reframe Space, Play, and the Ordinary. Critical Studies in Media Communication, 25(5), 447-465. https://doi.org/10.1080/15295030802468081

Stenros, J. (2012). In defence of a magic circle: the social and mental boundaries of play. Proceedings of DiGRA Nordic 2012 Conference: Local and Global-Games in Culture and Society (Vol. 2012). https://doi.org/10.26503/todigra.v1i2.10

SPIN Unit. (2015). ImageTagger [MacOS, Windows]. SPIN Unit.

Thorne, S. (2000). Data analysis in qualitative research. Evidence-Based Nursing, 3(3), 68-70. https://doi.org/10.1136/ebn.3.3.68

Tifentale, A., \& Manovich, L. (2015). Selfiecity: Exploring photography and self-fashioning in social media. In Postdigital Aesthetics (pp. 109-122). Springer.

Vella, K., Johnson, D., Cheng, V. W. S., Davenport, T., Mitchell, J., Klarkowski, M., \& Phillips, C. (2017). A Sense of Belonging: Pokémon GO and Social Connectedness. Games and Culture, 1555412017719973. https://doi.org/10.1177/1555412017719973

Wang, D., Wu, T., Wen, S., Liu, D., Xiang, Y., Zhou, W., ... Alelaiwi, A. (2018). Pokémon GO in Melbourne CBD: A case study of the cyber-physical symbiotic social networks. Journal of Computational Science, 26, 456-467.

Wong, J., Balmford, W., Hjorth, L., \& Richardson, I. (2019). Asian International Students and Gaming Cultures in Melbourne: Play Co-presence and Place. In Video Games and the Global South (pp. 978-0-359-64139-0). Pittsburgh, PA, US: ETC Press.

Yang, C., \& Liu, D. (2017). Motives matter: motives for playing Pokémon Go and implications for well-being. Cyberpsychology, Behavior, and Social Networking, 20(1), 52-57. https://doi.org/10.1089/cyber.2016.0562 